\title{
Convergence of the hyperspherical-harmonics expansion with increasing number of particles for bosonic systems
}

\author{
N. K. Timofeyuk \\ Faculty of Electronics and Physical Sciences, Physics Department, University of Surrey, Guildford, Surrey GU2 7XH, United Kingdom
}

(Received 30 July 2012; published 24 September 2012)

\begin{abstract}
A formal proof is given that for $N$-boson systems in which two-body interaction potentials are described by a single Gaussian the ratio of higher-order to the lowest-order hyper-radial potentials decreases at $N \rightarrow \infty$ as $N^{-1 / 2}$ or faster. As a result, for such potentials, the convergence of binding energies for ground and several lowest excited states, obtained in expansion of the $N$-body wave function over the hyperspherical-harmonics basis, improves with increasing number of bosons. For a phenomenological three-body repulsive potential, introduced to account for the missing hard core, the ratio of higher-order to lowest-order hyper-radial potential, corresponding to this three-body potential, also decreases as $N^{-1 / 2}$ or faster when $N \rightarrow \infty$. Although adding the three-body contributions leads to increased influence from the total nondiagonal couplings around the node of the lowest hyper-radial potential, the arguments are given that this should not dramatically deteriorate the convergence if the range of repulsion is properly chosen. This means that the hyperspherical-harmonics expansion with soft two-body and repulsive three-body effective forces may become an attractive tool for studying the spectra of many-body systems. It is suggested that fine tuning of the three-body repulsion to reproduce the binding energies over a large region of $N$ is possible. In particular, it has been shown that an $N$-independent choice for the three-body repulsion exists for which the ground-state binding energies of $N \leqslant 112$ atoms of helium, obtained in the lowest-order approximation of the hyperspherical-harmonics expansion, are close to the prediction of the Green's function Monte Carlo method with a hard-core He-He potential.
\end{abstract}

DOI: 10.1103/PhysRevA.86.032507

PACS number(s): 31.15.xj, 31.15.xt

\section{INTRODUCTION}

The interaction potentials between two atoms are known to have a strong repulsive core due to their complex internal structure. This repulsive core creates difficulties in studying the systems, made of many atoms, from first principles. Because of this core the solutions of the many-body Schrödinger equation obtained in various variational approaches by expansions over some sets of basis functions converge slowly. In particular, slow convergence of the expansion over hypersphericalharmonics basis for three- and four-body systems when the hard core is present has been known for a very long time [1].

In two recent publications [2,3], aimed at studying universal relations between different binding energies in helium clusters within the hyperspherical-harmonics expansion, it has been noticed that for weakly bound systems these relations do not depend on the details of the two-body interactions but only on the masses of the atoms. Therefore, in Refs. [2,3] the hard-core He-He potential has been replaced by a soft potential that reproduces the helium dimer binding energy, the scattering length, and effective range predicted by calculations with hard-core potentials. However, using only this soft twobody potential overbinds the helium trimer and other helium clusters since repulsion at short He-He distances is missing. To account for this repulsion, a hypercentral three-body potential has been phenomenologically introduced. This potential was represented by a single Gaussian and its range and depth were adjusted to fit the helium trimer binding energy. The calculations performed with this potential for four, five, and six atoms of helium gave very similar binding energies to those obtained with the hard-core He-He potential in other ab initio approaches in Refs. [4,5].
It has been noticed in Ref. [2] that the convergence of the hyperspherical expansion for the soft phenomenological potentials improves both for the ground state and the first excited state when the number of helium atoms increases from four to six. This observation has motivated the investigation carried out in the present paper with the aim to find out if the improvement of convergence can be expected to continue with a further increasing of the number of helium atoms.

The convergence of the ground-state binding energy depends on how strong the couplings between the lowest- and higher-order terms of hyperspherical-harmonics expansion are. Therefore, the main focus in the present paper is the ratio of the higher-order and the lowest-order hyper-radial potentials for bosonic systems. This ratio is studied using a specific presentation of hyperspherical harmonics via the symmetrized products of oscillator single-particle wave functions [6,7], as explained in Sec. II. With this method the matrix elements in the hyperspherical basis can be calculated easily for any number of particles $N$. The asymptotic expression for the ratio between the lowest- and other-order hyper-radial potentials for $N \rightarrow \infty$ is derived in Sec. III for two-body potentials described by a single Gaussian. It proves the decrease of the relative strength of the nondiagonal couplings, thus explaining the observations of improved convergence in Ref. [2] for $N=5$ and $N=6$. In Sec. III B further improvement of the convergence is demonstrated for $N=8,10$, and 20 for the same potential. Section III C discusses the qualitative changes in convergence for potentials described by two Gaussians and gives a few examples of the binding energy convergence. The hypercentral three-body force is introduced in Sec. IV. The leading contributions to the hyper-radial potentials for a large number of atoms and the ratio between the nondiagonal to diagonal hyper-radial potentials related to this force are derived 
and the consequence for convergence is discussed. Section V discusses the possibility of fine tuning the three-body force and draws conclusions.

\section{HYPERSPHERICAL FORMALISM}

In the present paper, it is assumed that the internal spin of bosons is $0^{+}$and that the total spin of the $N$-boson systems is $0^{+}$as well.

\section{A. Hyperspherical expansion and radial equations}

In the hyperspherical-harmonics method [8], the $N$-body wave function $\Psi_{N}$ is a function of a vector $\rho$ in a $3(N-1)$ dimensional space formed by $N-1$ Jacobi coordinates $\xi_{i}=$ $\sqrt{i /(i+1)}\left(\sum_{j=1}^{i} \boldsymbol{r}_{j} / i-\boldsymbol{r}_{i+1}\right)$. In this space, the hyperspherical coordinates $(\rho, \hat{\rho})$ are introduced, where the hyper-radius $\rho$ is the square of the length of $\rho$,

$$
\rho^{2}=\sum_{i=1}^{N-1} \boldsymbol{\xi}_{i}^{2}=\sum_{i=1}^{N} \boldsymbol{r}_{i}^{2}-\boldsymbol{R}^{2}=\frac{1}{N} \sum_{i<j}^{N}\left(\boldsymbol{r}_{i}-\boldsymbol{r}_{j}\right)^{2},
$$

$\boldsymbol{r}_{i}$ are individual coordinates of bosons, and $\boldsymbol{R}=$ $\left(\sum_{i=1}^{N} \boldsymbol{r}_{i}\right) / \sqrt{N}$ is the normalized coordinate of the center of mass. The rest of the $3 N-4$ variables are the hyperangles $\hat{\rho} \equiv\left\{\theta_{1}, \theta_{2}, \ldots, \theta_{3 N-4}\right\}$ but their choice is not important in the approach used in the present work.

The wave function $\Psi_{N}(\rho, \hat{\rho})$ is expanded onto a complete set of eigenfunctions $Y_{K \gamma}(\hat{\rho})$ of the angular part of the $3 N-4$ dimensional Laplacian $\Delta_{\hat{\rho}}[8]$

$$
\Delta_{\hat{\rho}} Y_{K \gamma}(\hat{\rho})=K(K+3 N-5) Y_{K \gamma}(\hat{\rho}) .
$$

The quantum number $K$ is a hyperangular momentum and the index $\gamma$ denotes the set of all other possible quantum numbers. The hyperspherical harmonics $Y_{K \gamma}(\hat{\rho})$ are constructed to be symmetric with respect to permutations of identical boson coordinates. The hyperspherical-harmonics expansion then reads

$$
\Psi_{N}(\rho, \hat{\rho})=\rho^{-(3 N-4) / 2} \sum_{K \gamma} \chi_{K \gamma}(\rho) Y_{K \gamma}(\hat{\rho}) .
$$

For total spin and parity $0^{+}$the value $K$ runs from 0 to infinity with the step of 2 excluding $K=2$ for which symmetric hyperharmonics do not exist. The hyper-radial functions $\chi_{K \gamma}(\rho)$ are found from the solution of the coupled set of differential equations

$$
\begin{aligned}
& \left(\frac{d^{2}}{d \rho^{2}}-\frac{\mathcal{L}_{K}\left(\mathcal{L}_{K}+1\right)}{\rho^{2}}-\frac{2 m}{\hbar^{2}}\left[E+V_{K \gamma, K \gamma}(\rho)\right]\right) \chi_{K \gamma}(\rho) \\
& =\frac{2 m}{\hbar^{2}} \sum_{K^{\prime} \gamma^{\prime} \neq K \gamma} V_{K \gamma, K^{\prime} \gamma^{\prime}}(\rho) \chi_{K^{\prime} \gamma^{\prime}}(\rho),
\end{aligned}
$$

where $\mathcal{L}_{K}=K+(3 N-6) / 2, m$ is the boson mass and the hyper-radial potentials $V_{K \gamma, K^{\prime} \gamma^{\prime}}(\rho)$ are the matrix elements of the two-body interactions,

$$
V_{K \gamma, K^{\prime} \gamma^{\prime}}(\rho)=\left\langle Y_{K \gamma}(\hat{\rho})\left|\sum_{i<j} V_{i j}\left(\boldsymbol{r}_{i}-\boldsymbol{r}_{j}\right)\right| Y_{K^{\prime} \gamma^{\prime}}(\hat{\rho})\right\rangle .
$$

Three-body and any other interactions can also be added when necessary.

\section{B. Construction of hyperspherical harmonics}

The hyperspherical harmonics $Y_{K \gamma}(\hat{\rho})$ are constructed here using the method developed in Refs. [6,7], in which

$$
Y_{K \gamma}(\hat{\rho})=\Psi_{0 K \gamma}(\rho, \hat{\rho}) / R_{0 K}(\rho) .
$$

Here, $\Psi_{\kappa K \gamma}(\rho, \hat{\rho})$ is an eigenfunction of the the Schrödinger equation with the two-body potential $V_{i j}=\omega r_{i j}^{2}$, for which exact solutions are known, and $R_{\kappa K}(\rho)$ is the hyper-radial wave function [8]

$$
\begin{aligned}
R_{\kappa K}(\rho)= & b^{-n / 2}\left(\frac{2 \kappa !}{\Gamma(\kappa+K+n / 2)}\right)^{1 / 2}\left(\frac{\rho}{b}\right)^{K} \\
& \times L_{\kappa}^{K+(n-2) / 2}\left(\rho^{2} / b^{2}\right) e^{-\rho^{2} / 2 b^{2}},
\end{aligned}
$$

with $b=\sqrt{\hbar / m \omega}$ being the oscillator radius and $n=3 N-3$. The number of nodes $\kappa$ is set to zero for practical purposes as discussed in Ref. [6]. The right-hand side of Eq. (6) is multiplied above and below by the $0 s$ center-of-mass wave function $\Phi_{000}(\boldsymbol{R})$ and the product $\Psi_{0 K \gamma}(\rho, \hat{\rho}) \Phi_{000}(\boldsymbol{R})$ in the nominator is expanded onto the linear combination of the symmetrized products $\Phi_{K i}\left(\boldsymbol{r}_{1}, \boldsymbol{r}_{2}, \ldots, \boldsymbol{r}_{N}\right)$ of the singleparticle oscillator wave functions $\phi_{\alpha}\left(\boldsymbol{r}_{i}\right)$ with the total number of oscillator quanta equal to $K$. Therefore, the hyperharmonics $Y_{K \gamma}(\hat{\rho})$ can be written as

$$
Y_{K \gamma}(\hat{\rho})=\frac{\sum_{i} C_{i}^{K \gamma} \Phi_{K i}\left(\boldsymbol{r}_{1}, \boldsymbol{r}_{2}, \ldots, \boldsymbol{r}_{N}\right)}{\Phi_{000}(\boldsymbol{R}) R_{0 K}(\rho)} .
$$

The individual coordinates $\boldsymbol{r}_{i}$ are chosen in an arbitrary fixed coordinate system independent of the center-of-mass motion and the coefficients $C_{i}^{K \gamma}$ are chosen in such a way that $Y_{K \gamma}(\hat{\rho})$ does not depend on the choice of the origin of the coordinate system, nor on the hyper-radius $\rho$ or the oscillator radius $b$. To achieve this and to provide a well-defined angular momentum of the system, the expansion coefficients $C_{i}^{K \gamma}$ are found as the eigenvectors of three matrices: the matrix $\boldsymbol{J}^{2}$ of the total spin, the matrix $\boldsymbol{R}^{2}$ of the center-of-mass radius, and the matrix of the $3 A-4$-dimensional hyperangular Laplacian $\Delta_{\hat{\rho}}$ rewritten in individual coordinates $\left\{\boldsymbol{r}_{i}\right\}$. These eigenvectors correspond to the eigenvalues $J(J+1), \frac{3}{2} b^{2}$ and $K(K+n-2)$ of the matrices $\boldsymbol{J}^{2}, \boldsymbol{R}^{2}$, and $\Delta_{\hat{\rho}}$, respectively (see Ref. [7] for more details).

\section{Hyper-radial potentials}

The calculated coefficients $C_{i}^{K \gamma}$ are used to obtain the hyper-radial potentials $V_{K \gamma, K^{\prime} \gamma^{\prime}}(\rho)$ that enter Eq. (4),

$$
V_{K \gamma, K^{\prime} \gamma^{\prime}}(\rho)=\sum_{i i^{\prime}} C_{i}^{K \gamma} C_{i^{\prime}}^{K^{\prime} \gamma^{\prime}} V_{K i, K^{\prime} i^{\prime}}(\rho) .
$$

$V_{K i, K^{\prime} i^{\prime}}(\rho)$ are related to the matrix elements $\left\langle\Phi_{K^{\prime} i^{\prime}}\left|\sum_{i<j} V_{i j}\right| \Phi_{K i}\right\rangle$ in the oscillator basis $\left|\Phi_{K i}\right\rangle$ by the inverse Laplace transform [6]

$$
\begin{aligned}
V_{K i, K^{\prime} i^{\prime}}(\rho)= & \frac{\left(\Gamma(K+n / 2) \Gamma\left(K^{\prime}+n / 2\right)\right)^{1 / 2}}{\rho^{K+K^{\prime}+n-2}} \frac{1}{2 \pi i} \int_{-i \infty}^{i \infty} d s \\
& \times e^{s \rho^{2}} s^{-\left(K+K^{\prime}+n\right) / 2}\left\langle\Phi_{K^{\prime} i^{\prime}}\left|\sum_{i<j} V_{i j}\right| \Phi_{K i}\right\rangle, \quad(10)
\end{aligned}
$$

in which $s=b^{-2}$ and the integration path bypasses the origin in the counterclockwise direction. 


\section{LOWEST-ORDER APPROXIMATION AND ITS COUPLING TO HIGHER-ORDER TERMS}

\section{A. Lowest-order $\boldsymbol{K}=\mathbf{0}$ approximation for Gaussian potentials}

In the lowest-order approximation, $K=0$, only one term is retained in the expansion (3) with the radial function $\chi_{0}(\rho)$ satisfying the equation

$$
\left[\frac{d^{2}}{d \rho^{2}}-\frac{L(L+1)}{\rho^{2}}-\frac{2 m}{\hbar^{2}}\left[E+V_{00}(\rho)\right]\right] \chi_{0}(\rho)=0 .
$$

The hyper-radial potential $V_{00}(\rho)$ is evaluated from Eq. (10) in which the matrix element $\left\langle\Phi_{00}\left|\sum_{i<j} V_{i j}\right| \Phi_{00}\right\rangle$ is replaced with $\frac{N(N-1)}{2}\left\langle\Phi_{00}\left|V_{N, N-1}\right| \Phi_{00}\right\rangle$. The function $\Phi_{00}$ is just a product $\phi_{00}\left(\boldsymbol{r}_{1}\right) \phi_{00}\left(\boldsymbol{r}_{2}\right) \cdots \phi_{00}\left(\boldsymbol{r}_{N}\right)$ of the single-particle oscillator $0 s$ wave functions $\phi_{00}(\boldsymbol{r})=2\left(s^{3} / \pi\right)^{1 / 4} e^{-s r^{2} / 2} Y_{00}(\hat{\boldsymbol{r}})$, where $Y$ is the spherical harmonics.

Assuming the Gaussian shape of the two-body potential,

$$
V_{i j}(r)=V_{0} e^{-(r / \alpha)^{2}}
$$

we find that

$$
\left\langle\Phi_{00}\left\|V_{N, N-1}\right\| \Phi_{00}\right\rangle=V_{0}\left(1+\frac{2}{s \alpha^{2}}\right)^{-3 / 2}
$$

and, after integration over $d s$ in Eq. (10),

$$
V_{00}(\rho)=\frac{N(N-1)}{2} V_{0} M\left(\frac{3}{2}, \frac{n}{2},-\frac{2 \rho^{2}}{\alpha^{2}}\right),
$$

where $M$ is the confluent hypergeometric function. Asymptotically, $V_{00}(\rho)$ decreases as $\rho^{-3}$.

\section{B. Coupling between the lowest-order and other hyperspherical harmonics}

The hyperharmonics of the order $K$ are obtained from all possible $N$-body oscillator wave functions $\Phi_{K i}$, where $i=\left\{v_{1} l_{1} m_{1} ; \ldots ; v_{N} l_{N} m_{N}\right\}, v_{j}$ is the number of nodes, and $l_{j}\left(m_{j}\right)$ is the orbital momentum (its projection) of the single-particle state of the $j$ th boson, $\sum_{i=1}^{N}\left(2 v_{i}+l_{i}\right)=K$ and, in addition, $\sum_{i=1}^{N} m_{i}=0$ for zero total spin of the system. However, only the sets $i$ of the type $(0 s)^{N-2}\left(v_{1} l_{1}\right)\left(v_{2} l_{2}\right)$, where $2 v_{1}+l_{1}+2 v_{2}+l_{2}=K$, will give nonzero contributions to the nondiagonal hyper-radial potentials

$$
\begin{aligned}
V_{00, K i}(\rho)= & \frac{N(N-1)}{2} \frac{\left(\Gamma\left(\frac{n}{2}\right) \Gamma\left(\frac{n}{2}+K\right)\right)^{1 / 2}}{\rho^{n+K-2}} \frac{1}{2 \pi i} \int_{-i \infty}^{i \infty} d s \\
& \times e^{s \rho^{2}} s^{-(n+K) / 2}\left\langle\Phi_{0}\left|V_{N, N-1}\right| \Phi_{K i}\right\rangle .
\end{aligned}
$$

For these $i$,

$$
\left\langle\Phi_{0}\left|V_{N, N-1}\right| \Phi_{K i}\right\rangle=a_{i}(N) c_{i}(K)\left\langle\phi_{00}|| V\left(\sqrt{2} r_{N, N-1}\right)|| \phi_{\nu 0}\right\rangle,
$$

where $v=K / 2$. The coefficients $a_{i}(N)$ arise because all exchange terms in $\Phi_{K i}$ but one give zero contribution to the matrix element $\left\langle\Phi_{0}\left|V_{N, N-1}\right| \Phi_{K i}\right\rangle$. They are equal to

$$
\begin{aligned}
a_{i}(N) & =2 / \sqrt{N}, \quad 2 v_{1}+l_{1}=0, \\
& =\frac{1+\delta_{v_{1} v_{2}} \delta_{l_{1} l_{2}}}{\sqrt{N(N-1) / 2}}, \quad 2 v_{1}+l_{1} \neq 0,
\end{aligned}
$$

TABLE I. Coefficients $c_{i}(K)$ for $K=4$.

\begin{tabular}{lc}
\hline \hline$i$ & $c_{i}$ \\
\hline$(0 s)^{N-1} 2 s$ & $1 / 4$ \\
$(0 s)^{N-2}(0 d)^{2}$ & $1 / \sqrt{6}$ \\
$(0 s)^{N-2} 0 p 1 p$ & $-1 / 2$ \\
$(0 s)^{N-2}(1 s)^{2}$ & $\sqrt{5 / 24}$ \\
\hline \hline
\end{tabular}

thus carrying an explicit dependence on the number of bosons. They decrease with $N$ because more exchange terms in $\Phi_{K i}$ become available. As for the coefficients $c_{i}(K)$, they are the standard Talmi-Moshinski brackets $\left\langle\nu 0,00: 0|1: 1| v_{1} l_{1}, v_{2} l_{2}: 0\right\rangle$ for transforming the product of the single-particle wave functions $\left|\phi_{v_{1} l_{1}}\left(\boldsymbol{r}_{N-1}\right) \phi_{v_{2} l_{2}}\left(\boldsymbol{r}_{N}\right): 0\right\rangle$ into the oscillator wave functions in the relative $\boldsymbol{x}=\left(\boldsymbol{r}_{N-1}-\boldsymbol{r}_{N}\right) / \sqrt{2}$ and the twobody center-of-mass $\boldsymbol{X}=\left(\boldsymbol{r}_{N-1}+\boldsymbol{r}_{N}\right) / \sqrt{2}$ coordinates [9]. These coefficients are always smaller than one and they do not depend on $N$. Examples of $c_{i}(K)$ for the next-to-lowest-order harmonics, $K=4$, are given in Table I. For $i=(0 s)^{N-1}(v s)$ the coefficients $c_{i}(K)$ are equal to $(-1)^{K} / 2^{K / 2}$.

Using the single-particle oscillator wave functions

$$
\phi_{\nu l}(r)=\sqrt{\frac{2 v !}{\Gamma(v+l+3 / 2)}} s^{(2 l+3) / 4} r^{l} e^{-s r^{2} / 2} L_{v}^{l+1 / 2}\left(s r^{2}\right),
$$

we can evaluate the matrix element in Eq. (16):

$$
\begin{aligned}
& \left\langle\phi_{00}\left\|V\left(\sqrt{2} r_{N, N-1}\right)\right\| \phi_{\nu 0}\right\rangle \\
& =\sqrt{\frac{\Gamma\left(v+\frac{3}{2}\right)}{v ! \Gamma\left(\frac{3}{2}\right)}} V_{0}\left(\frac{2}{s \alpha^{2}}\right)^{v}\left(1+\frac{2}{s \alpha^{2}}\right)^{-v-3 / 2} .
\end{aligned}
$$

Then integrating over $d s$ in Eq. (15) we get for $v=K / 2$

$$
\begin{aligned}
V_{00, K i}(\rho)= & a_{i}(N) c_{i}(K) \sqrt{\frac{\Gamma\left(\frac{n}{2}\right) \Gamma\left(\frac{K+3}{2}\right)}{\Gamma\left(\frac{3}{2}\right) \Gamma\left(\frac{K}{2}+1\right) \Gamma\left(\frac{n}{2}+K\right)}} \\
& \times \frac{N(N-1)}{2} V_{0}\left(\frac{2 \rho^{2}}{\alpha^{2}}\right) \\
& \times M\left(\frac{K+3}{2} ; \frac{n}{2}+K ;-\frac{2 \rho^{2}}{\alpha^{2}}\right) .
\end{aligned}
$$

The confluent hypergeometric function $M$ can be presented as

$M\left(\frac{K+3}{2} ; \frac{n}{2}+K ;-\frac{2 \rho^{2}}{\alpha^{2}}\right)=\frac{\Gamma\left(\frac{n}{2}+K\right)}{\Gamma\left(\frac{n+K-3}{2}\right) \Gamma\left(\frac{K+3}{2}\right)} I_{n, K}(\rho)$,

where

$$
I_{n, K}(\rho)=\int_{0}^{1} d t e^{-2 \rho^{2} t / \alpha^{2}} t^{(K+1) / 2}(1-t)^{(n+K-5) / 2} .
$$

Using Eqs. (14), (20), and (21) the ratio $U_{K i}(\rho)$ of the nondiagonal to diagonal hyper-radial potential can be constructed:

$$
\begin{gathered}
U_{K i}(\rho) \equiv \frac{V_{00, K i}(\rho)}{V_{00,00}(\rho)}=a_{i}(N) c_{i}(K) \sqrt{\frac{2^{K} \Gamma\left(\frac{n}{2}+K\right)}{\Gamma\left(\frac{n}{2}\right) \Gamma(K+2)}} \\
\quad \times \frac{\Gamma\left(\frac{n-3}{2}\right)}{\Gamma\left(\frac{n+K-3}{2}\right)}\left(\frac{2 \rho^{2}}{\alpha^{2}}\right)^{K / 2} \frac{I_{n, K}(\rho)}{I_{00}(\rho)} .
\end{gathered}
$$




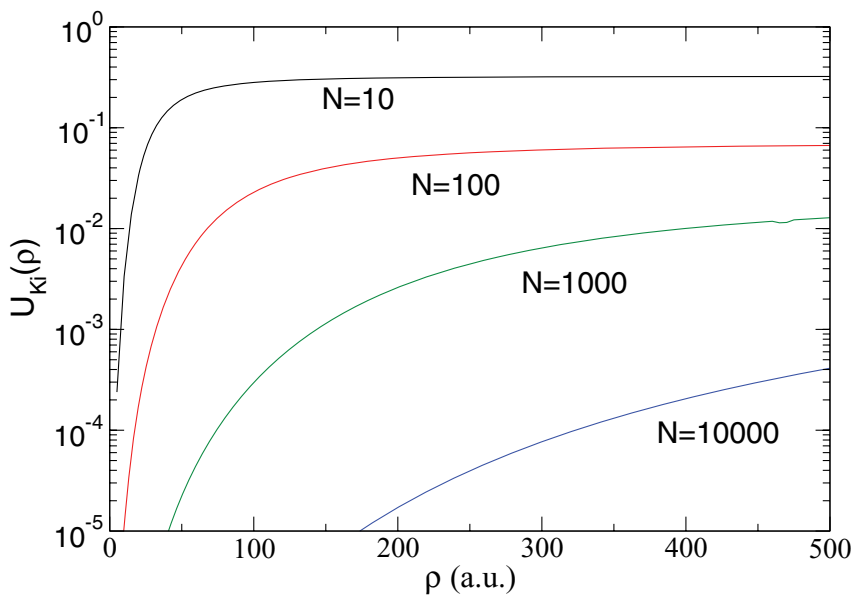

FIG. 1. (Color online) Upper limit for the ratio $U_{K i}(\rho)$ of nondiagonal to diagonal hyper-radial potential as a function of $\rho$ calculated for $K=4$ and $i=(0 s)^{N-1}(v s)$. The range $\alpha=10.03$ a.u. of the two-body potential has been used.

As $N \rightarrow \infty$, and therefore as $n \rightarrow \infty$, this ratio tends to

$$
U_{K i}(\rho) \rightarrow a_{i}(N) c_{i}(K) \sqrt{\frac{2^{K}}{\Gamma(K+2)}}\left(\frac{2 \rho^{2}}{\alpha^{2}}\right)^{K / 2} \frac{I_{n, K}(\rho)}{I_{00}(\rho)} .
$$

Since the integrands of both $I_{n, K}(\rho)$ and $I_{00}(\rho)$ are positive and the integrand of $I_{n, K}(\rho)$ is obtained from the integrand of $I_{00}(\rho)$ by multiplying the latter by the function $t^{K / 2}(1-t)^{K / 2}$ which is smaller than one over the interval $0 \leqslant t \leqslant 1$, then $I_{n, K}(\rho)<$ $I_{00}(\rho)$ for all $\rho$ and $N$. Thus the ratio $U_{K i}(\rho)$ should decrease with large $N$. The rate of decrease, however, depends on $\rho$. As $\rho \rightarrow 0$ the ratio of the two hypergeometric functions in $V_{00, K i}$ and $V_{00}$ is one so that $U_{K i}(\rho)$ tends to zero and decrease with $N$ as $a_{i}(N) N^{-K / 2}$. As $\rho \rightarrow \infty, U_{K i}(\rho) \rightarrow a_{i}(N)$ when $N \rightarrow$ $\infty$, which is obtained using asymptotic expressions for the confluent hypergeometric functions. For finite $\rho$ the decrease of $U_{K i}(\rho)$ with $N$ is not as fast as for $\rho \rightarrow 0$ but not as slow as for $\rho \rightarrow \infty$. This is demonstrated in Fig. 1, where $U_{K i}(\rho)$ are shown as functions of $\rho$ for several values of $N$ for $K=4$ and $i=(0 s)^{N-1}(2 s)$, for which decrease with $N$ is expected to be the slowest. The $U_{K i}(\rho)$ were calculated using $\alpha=10.03$ a.u.

Thus, we have proved that the contribution from an individual set $i$ to the hyper-radial potential $V_{00, K \gamma}$ decreases with $N$.
Next, it should be proved that $\sum_{i} C_{i}^{K \gamma} V_{00, K i} / V_{00}$ decreases with $N$ as well. It should be noted first that for any $K$ there is only one $i$ which gives the slowest decrease of $U_{K i}(\rho)$ with $N$, namely $i=(0 s)^{N-1}(v s)$, where $v=K / 2$. Then the contribution to $U_{K i}(\rho)$ from this $i, C_{i}^{K \gamma} V_{00, K i} / V_{00, K i}$, decreases with $N$ as well because $\left|C_{i}^{K \gamma}\right| \leqslant 1$. Other sets of $i$ that give nonzero terms in nondiagonal coupling potentials, namely, $i=$ $(0 s)^{N-2}\left(v_{1} l_{1}\right)\left(v_{2} l_{2}\right)$, at a fixed $\rho$ and $K$ give the contribution of $\left|\sum_{i} C_{i}^{K \gamma} V_{00, K i}(\rho)\right| \leqslant N_{i}(K) \max \left(\left|V_{00, K i}(\rho)\right|\right)$, where $N_{i}(K)$ is the number of sets $\left\{v_{1} l_{1}, v_{2} l_{2}\right\}$ with $2 v_{1}+l_{1}+2 v_{2}+l_{2}=$ $K$. Since $N_{i}(K)$ does not depend on $N$, the corresponding contribution $\sum_{i} C_{i}^{K \gamma} V_{00, K i}(\rho)$ decreases with $N$ at the same rate as $V_{00, K i}(\rho)$, which for these $i$ is $\sim 1 / \sqrt{N(N-1)}$ as $N \rightarrow$ $\infty$. Thus, the strength of nondiagonal coupling decreases with respect to the lowest-order term for Gaussian potentials when the number of bosons increases.

To demonstrate improved convergence with $N$ as the nondiagonal couplings become weaker, the ground-state energies for $N=8,10$, and 20 helium atoms were calculated with the soft He-He potential from Ref. [2], for which $V_{0}=-1.227 \mathrm{~K}$ and $\alpha=10.03$ a.u. Below, this potential is referred to as potential A. The resulting energies are shown in Table II for several $K_{\max }$ together with the energies for $N=4$ and $N=6$ from Ref. [2]. The $\hbar^{2} / m=43.281307$ (a.u. $)^{2} \mathrm{~K}$ value were used in these calculations, the same as in Ref. [2]. The hyper-radial potentials $V_{K \gamma, K^{\prime} \gamma^{\prime}}(\rho)$ were calculated using the code developed in Ref. [7] and the system of differential equations [Eq. (4)] was solved by expansion on a Sturmian basis using the core STURMXX [10]. The energies published in Ref. [2] for $A \leqslant 6$ are reproduced up to all five published digits.

\section{Two-Gaussian potentials}

In the case when the two-body potential is a sum of two Gaussians,

$$
V_{i j}(r)=V_{1} e^{-\left(r / \alpha_{1}\right)^{2}}+V_{2} e^{-\left(r / \alpha_{2}\right)^{2}}
$$

the hyper-radial potentials $V_{00, K i}(\rho)$ are determined by the sum $V_{00, K i}^{(1)}(\rho)+V_{00, K i}^{(2)}(\rho)$, the first term of which corresponds to $\left(V_{1}, \alpha_{1}\right)$ and the second one to $\left(V_{2}, \alpha_{2}\right)$. Let us assume that $\alpha_{1}>\alpha_{2}$ and that both $V_{1}$ and $V_{2}$ are negative. The ratio $U_{K i}(\rho)$

TABLE II. Absolute values of binding energies (in K) for a system of $N$ helium atoms obtained with different two-body potentials (see text) for several values of $K_{\max }$. The energies for $N=4$ and $N=6$ with set A are the same as in Ref. [2]. The fifth digit for energy of the

\begin{tabular}{|c|c|c|c|c|c|c|c|c|c|c|c|}
\hline \multirow[b]{2}{*}{$K_{\max }$} & \multicolumn{5}{|c|}{ Potential A } & \multicolumn{3}{|c|}{ Potential B } & \multicolumn{3}{|c|}{ Potential C } \\
\hline & $N=4$ & $N=6$ & $N=8$ & $N=10$ & $N=20$ & $N=4$ & $N=20$ & $N=200$ & $N=4$ & $N=20$ & $N=200$ \\
\hline 0 & 0.72598 & 3.7731 & 9.7159 & 18.855 & 117.81 & 2.3779 & 161.93 & 19931 & 0.7358 & 42.888 & 4989.5 \\
\hline 4 & 0.74645 & 3.8076 & 9.7607 & 18.908 & 117.89 & 2.4044 & 163.90 & 20114 & 1.0316 & 45.857 & 5117.7 \\
\hline 6 & 0.75015 & 3.8099 & 9.7623 & 18.909 & 117.89 & 2.4283 & 164.97 & 20162 & 1.3796 & 48.036 & 5162.3 \\
\hline 8 & 0.75106 & 3.8108 & 9.7632 & 18.910 & 117.89 & 2.4480 & 165.77 & 20250 & 1.6096 & 50.346 & 5223.4 \\
\hline 10 & 0.75128 & 3.8109 & 9.7633 & 18.910 & 117.89 & 2.4566 & 166.43 & 20299 & 1.6510 & 52.726 & 5266.7 \\
\hline 12 & 0.75135 & 3.8109 & 9.7633 & & & 2.4648 & 166.99 & & 1.6719 & 55.114 & \\
\hline 14 & 0.75137 & 3.8109 & & & & 2.4684 & & & 1.6767 & & \\
\hline
\end{tabular}
$N=20$ system obtained with $K_{\max }=20$ and potential C may be not sufficiently accurate. 
then becomes

$$
U_{K i}(\rho)=\frac{V_{00, K i}^{(1)}(\rho)}{V_{00}^{(1)}(\rho)} \frac{1+V_{00, K i}^{(2)}(\rho) / V_{00, K i}^{(1)}(\rho)}{1+V_{00}^{(2)}(\rho) / V_{00}^{(1)}(\rho)} .
$$

For $\alpha_{1}>\alpha_{2}, M\left(a, b ;-\frac{2 \rho^{2}}{\alpha_{1}^{2}}\right)>M\left(a, b ;-\frac{2 \rho^{2}}{\alpha_{1}^{2}}\right)$ for all values $a$ and $b$ that appear in the hyperspherical approach. Therefore, the ratio $V_{00, K i}^{(2)}(\rho) / V_{00, K i}^{(1)}(\rho)$, calculated using Eqs. (14) and (20), is restricted by $V_{2} \alpha_{1}^{K} /\left(V_{1} \alpha_{2}^{K}\right)$ so that $U_{K i}(\rho)$ is restricted as well,

$$
U_{K i}^{(1)}(\rho) \frac{1}{1+\frac{V_{2}}{V_{1}}} \leqslant U_{K i}(\rho) \leqslant U_{K i}^{(1)}(\rho)\left(1+\frac{V_{2}}{V_{1}} \frac{\alpha_{1}^{K}}{\alpha_{2}^{K}}\right)
$$

for all $N$. In Eq. (27), $U_{K i}^{(1)}(\rho) \equiv V_{00, K i}^{(1)}(\rho) / V_{00}^{(1)}(\rho)$. It has been shown above that $U_{K i}^{(1)}(\rho)$ decreases with $N$ at least as $N^{-1 / 2}$. Therefore, $U_{K i}(\rho)$ is also a decreasing function of $N$ and convergence of the hyperspherical expansion for a sum of two attractive Gaussian potentials should be similar to the convergence for one-Gaussian potential.

The situation is different when $V_{1}$ and $V_{2}$ have different signs. Then at some point $\rho_{0}, V_{00}\left(\rho_{0}\right)=0$ while $V_{00, K=4 i}\left(\rho_{0}\right) \neq 0$. At such points, even if individual $U_{K=4 i}^{(j)}(\rho)$ decrease with $N$, the total nondiagonal coupling $V_{00, K=4 i}\left(\rho_{0}\right)$ is infinitely larger than the lowest-order diagonal potential $V_{00}\left(\rho_{0}\right)$. Whether this influences the convergence of the hyperspherical-harmonics expansion depends on the relative strength of $V_{00, K=4 i}\left(\rho_{0}\right)$, the centrifugal potential $V_{\text {cent }}(\rho) \equiv$ $\frac{\hbar^{2}}{2 m} \mathcal{L}_{K}\left(\mathcal{L}_{K}+1\right) / \rho^{2}$ at $\rho=\rho_{0}$, and the total binding energy $E$. If $\left|V_{00, K=4 i}\left(\rho_{0}\right)\right| \ll V_{\text {cent }}\left(\rho_{0}\right)$, the convergence of the hyperspherical expansion should not deteriorate much whether the binding energy $E$ is large or small. If $\left|V_{00, K=4 i}\left(\rho_{0}\right)\right|$ is comparable or larger than $V_{\text {cent }}\left(\rho_{0}\right)$ then convergence over $K$ for a fixed $N$ will be determined by how small the $V_{00, K=4 i}\left(\rho_{0}\right)$ are with respect to the binding energy $E$. For $V_{00, K=4 i}\left(\rho_{0}\right) \sim E$ or $V_{00, K=4 i}\left(\rho_{0}\right)>E$ the convergence should be poor. However, $E$ increases with $N$ and at some point where $E$ becomes much larger than the largest nondiagonal coupling $V_{00, K=4 i}\left(\rho_{0}\right)$ the convergence should improve. To illustrate this idea, the binding energies for $N=4,20$, and 200 were calculated with two potentials, (B) $V(r)=-2.227 e^{-(r / 10.03)^{2}}+12.3 e^{-(r / 2)^{2}}$ and $(\mathrm{C})$ $V(r)=-5.134 e^{-(r / 10.03)^{2}}+20 e^{-(r / 5)^{2}}$, both of which give the same He-He binding energy of $-1.296 \mathrm{mK}$ as the potential A from Ref. [2]. The potential B produces for $N=4$ a node in the hyper-radial potential $V_{00}(\rho)$ where the centrifugal potential $V_{\text {cent }}\left(\rho_{0}\right)$ significantly larger than $V_{00, K=4 \gamma}\left(\rho_{0}\right)$ [see Fig. 2(a)]; however, $V_{00, K=4 \gamma}(\rho)$ has a noticeable tail at large $\rho$. The resulting binding energy changes very slowly with increasing $K_{\max }$ (see Table II). The convergence for $N=20$ and 200 is better because $V_{00, K=4 \gamma}\left(\rho_{0}\right)$ becomes smaller with respect to $E$. As for potential $\mathrm{C}$, it gives for $N=4$ a strong nondiagonal potential $V_{00, K=4 \gamma}(\rho)$ at $\rho_{0}$, larger than both the centrifugal potential and the energy $E$ [see Fig. 2(b)]. As a result, the convergence of the hyperspherical expansion for this potential deteriorates (see Table II). The same is true for $N=20$, although the difference between the two nearest energies becomes smaller. As $V_{00, K=4 \gamma}\left(\rho_{0}\right)$ decreases with respect to $E$ with increasing $N$ [Fig. 2(c)], the convergence of the binding energy improves. This is clearly seen for $N=200$ calculations with potential C.
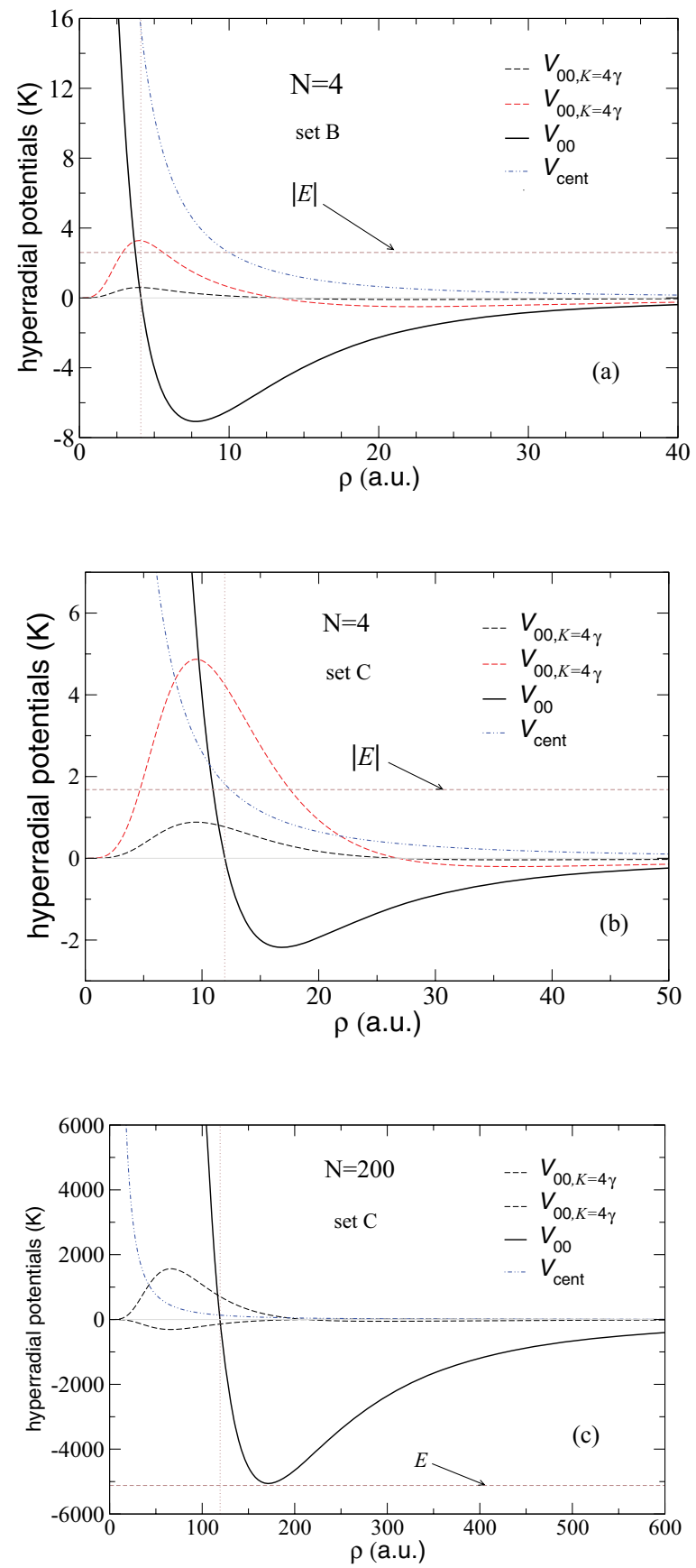

FIG. 2. (Color online) Two nondiagonal hyper-radial potentials $V_{00, K=4 \gamma}(\rho)$ in comparison to the lowest-order potential $V_{00}(\rho)$, centrifugal potential $V_{\text {cent }}$, and the ground-state energy $E$ for $N=4$ (a),(b) and $N=200$ (c) atoms of helium calculated with two sets of the He-He potential.

\section{LOWEST-ORDER APPROXIMATION WITH HYPERCENTRAL THREE-BODY FORCE AND ITS COUPLING TO THE HIGHER-ORDER TERMS}

\section{A. Lowest-order approximation}

For hypercentral three-body force

$$
W\left(\rho_{i j k}\right)=W_{0} e^{-2 \rho_{i j k}^{2} / \beta^{2}},
$$

where $\rho_{i j k}=\frac{2}{3}\left[\left(\boldsymbol{r}_{i}-\boldsymbol{r}_{j}\right)^{2}+\left(\boldsymbol{r}_{j}-\boldsymbol{r}_{k}\right)^{2}+\left(\boldsymbol{r}_{i}-\boldsymbol{r}_{k}\right)^{2}\right]$ is the three-body hyper-radius, the hyper-radial potential $W_{00}(\rho)$ of 
the $N$-body system is related to the three-body oscillator matrix element $\left\langle\Phi_{00}\left|W_{N-2, N-1, N}\right| \Phi_{00}\right\rangle$ :

$$
\begin{aligned}
W_{00}(\rho)= & \frac{N !}{3 !(N-3) !} \frac{\Gamma\left(\frac{n}{2}\right)}{\rho^{n-2}} \frac{1}{2 \pi i} \int_{-i \infty}^{i \infty} d s \\
& \times e^{s \rho^{2}} s^{-n / 2}\left\langle\Phi_{00}\left|W_{N-2, N-1, N}\right| \Phi_{00}\right\rangle .
\end{aligned}
$$

To calculate this matrix element, new variables, the normalized Jacobi coordinates are introduced:

$$
\begin{aligned}
& \boldsymbol{x}_{1}=\left(\boldsymbol{r}_{N-2}-\boldsymbol{r}_{N-1}\right) / \sqrt{2}, \\
& \boldsymbol{x}_{2}=\sqrt{\frac{2}{3}}\left[\left(\boldsymbol{r}_{N-2}+\boldsymbol{r}_{N-1}\right) / 2-\boldsymbol{r}_{N}\right], \\
& \boldsymbol{x}_{3}=\left(\boldsymbol{r}_{N-2}+\boldsymbol{r}_{N-1}+\boldsymbol{r}_{N}\right) / \sqrt{3} .
\end{aligned}
$$

Then using the relations

$$
\begin{gathered}
r_{N}^{2}+r_{N-1}^{2}+r_{N-2}^{2}=x_{1}^{2}+x_{2}^{2}+x_{3}^{2}, \\
r_{N, N-1}^{2}+r_{N-1, N-2}^{2}+r_{N-2, N}^{2}=3 x_{1}^{2}+3 x_{2}^{2},
\end{gathered}
$$

we get

$$
\begin{aligned}
\left\langle\Phi_{00}\left|W_{N-2, N-1, N}\right| \Phi_{00}\right\rangle & =W_{0}\left\langle\phi_{00}(x)\left|e^{-4 x^{2} / \beta^{2}}\right| \phi_{00}(x)\right\rangle^{2} \\
& =W_{0}\left(1+\frac{4}{s \beta^{2}}\right)^{-3}
\end{aligned}
$$

The hyper-radial potential then becomes

$$
W_{00}(\rho)=\frac{N !}{3 !(N-3) !} W_{0} M\left(3 ; \frac{n}{2} ;-\frac{4 \rho^{2}}{\beta^{2}}\right) .
$$

As $\rho \rightarrow \infty, W_{00}(\rho)$ decrease as $\rho^{-6}$, faster than the $\rho^{-3}$ decrease of the two-body potential $V_{00}(\rho)$.

\section{B. Coupling between the lowest-order and other hyperharmonics for three-body hypercentral potential}

The nondiagonal potential $W_{00, K \gamma}(\rho)$ for three-body potentials (28) are obtained in a similar way to the case of the two-body potentials,

$$
W_{00, K \gamma}(\rho)=\sum_{i i^{\prime}} C_{i}^{K \gamma} C_{i^{\prime}}^{K^{\prime} \gamma^{\prime}} W_{00, K i}(\rho),
$$

where

$$
\begin{aligned}
W_{00, K i}(\rho)= & \frac{N !}{3 !(N-3) !} \frac{\left[\Gamma\left(\frac{n}{2}\right) \Gamma\left(\frac{n}{2}+K\right)\right]^{1 / 2}}{\rho^{n+K-2}} \frac{1}{2 \pi i} \int_{-i \infty}^{i \infty} d s \\
& \times e^{s \rho^{2}} s^{-(n+K) / 2}\left\langle\Phi_{00}\left|W_{N-2, N-1, N}\right| \Phi_{K i}\right\rangle .
\end{aligned}
$$

Only the $i$ 's of the type $(0 s)^{N-3}\left(v_{1} l_{1} m_{1}\right)\left(v_{2} l_{2} m_{2}\right)\left(v_{3} l_{3} m_{3}\right)$, where $2 v_{1}+l_{1}+2 v_{2}+l_{2}+2 v_{3}+l_{3}=K$ and $m_{1}+m_{2}+$ $m_{3}=0$, give nonzero contributions to $W_{00, K i}(\rho)$. However, the slowest decrease with $N$ will come only from $i=(0 s)^{N-1}(\nu s)$, where $v=K / 2$, because of reasons similar to those discussed in Sec. III B. For this $i$

$$
\begin{aligned}
& \left\langle\Phi_{00}\left|W_{N-2, N-1, N}\right| \Phi_{K i}\right\rangle \\
& =(-)^{K}\left(\frac{2}{3}\right)^{\frac{K}{2}} \frac{3 W_{0}}{\sqrt{N}}\left\langle\phi_{00}(x)\right| e^{-4 x^{2} / \beta^{2}} \\
& \quad \times\left|\phi_{00}(x)\right\rangle\left\langle\phi_{00}(x)\left|e^{-4 x^{2} / \beta^{2}}\right| \phi_{\nu 0}(x)\right\rangle
\end{aligned}
$$

$$
\begin{aligned}
= & \frac{3(-)^{K} W_{0}}{\sqrt{N}} \sqrt{\frac{\Gamma\left(\frac{K+3}{2}\right)}{\Gamma\left(\frac{K}{2}+1\right) \Gamma\left(\frac{3}{2}\right)}} \\
& \times\left(\frac{8}{3 s \beta^{2}}\right)^{\frac{K}{2}}\left(1+\frac{4}{s \beta^{2}}\right)^{-3-\frac{K}{2}} .
\end{aligned}
$$

The coefficient $3 / \sqrt{N}$ comes from the normalization factor in the symmetrized wave function $\Phi_{K=4 i}$ and the number of exchange terms that give nonzero contribution to the matrix element $\left\langle\Phi_{00}\left|W_{N-2, N-1, N}\right| \Phi_{K i}\right\rangle$. The $(-)^{K}(2 / 3)^{K / 2}$ is the Talmi-Moshinsky transformation coefficient $\langle 0 s, 2 s: 0| 2$ : $1|0 s, 2 s: 0\rangle$ for particles with masses two and one. Substituting Eq. (37) into (36) and integrating over $d s$ we get

$$
\begin{aligned}
W_{00, K i}(\rho)= & \frac{N(N-1)(N-2) \frac{W_{0}}{\sqrt{N}}\left(-\sqrt{\frac{8}{3}} \frac{\rho}{\beta}\right)^{K}}{2} \\
& \times \sqrt{\frac{\Gamma\left(\frac{n}{2}\right) \Gamma\left(\frac{K+3}{2}\right)}{\Gamma\left(\frac{3}{2}\right) \Gamma\left(\frac{K}{2}+1\right) \Gamma\left(\frac{n}{2}+K\right)}} \\
& \times M\left(3+\frac{K}{2} ; \frac{n}{2}+K ;-\frac{4 \rho^{2}}{\beta^{2}}\right) .
\end{aligned}
$$

Following a reasoning similar to that of Sec. II B we can easily come to conclusion that $W_{00, K i}(\rho) / W_{00}(\rho)$ decreases at least as $N^{-1 / 2}$ in the $N \rightarrow \infty$ limit. However, it is the sum of the attractive two-body and repulsive three-body contributions that enters the Schrödinger equation. The convergence will, therefore, be determined by the strength of $V_{00, K \gamma}\left(\rho_{0}\right)+W_{00, K \gamma}\left(\rho_{0}\right)$ at the node $\rho_{0}$ of $V_{00}(\rho)+W_{00}(\rho)$. Once again, as in Sec. II C, if the nondiagonal couplings $V_{00, K \gamma}\left(\rho_{0}\right)+W_{00, K \gamma}\left(\rho_{0}\right)$ are comparable either to centrifugal or the total energy, the convergence of the hyperspherical expansion will be poor. If these couplings are much smaller than either $V_{\text {cent }}\left(\rho_{0}\right)$ or $E$ then the convergence should improve. At present, three-body potentials are not yet included in the code developed in Ref. [7] so the coupling potentials $V_{00, K \gamma}(\rho)+W_{00, K \gamma}(\rho)$ cannot be shown here and the $K>0$ calculations cannot yet been done. However, the contribution to these couplings that comes from $i=(0 s)^{N-1}(v s)$ is calculated for $K=4$ and results are presented in Fig. 3 for $N=10$ and 200. The calculations were performed with the two-body potential A and the three-body potential with $W_{0}=0.422 \mathrm{~K}$ and $\beta=14$ a.u. for which converged calculations for $N=4,5,6$ are given in Ref. [2].

For $N=10$, the hyper-radial potential $V_{00}(\rho)+W_{00}(\rho)$ does not have any nodes while the nondiagonal potential $V_{00, K=4 i}(\rho)+W_{00, K=4 i}(\rho)$ is much smaller than the diagonal one. Therefore, good convergence of the hyperspherical expansion should be expected. With increasing $N$, the repulsive three-body contributions increase as $\frac{N !}{(N-3) ! 3 !} \sim N^{3}$, faster than attractive two-body contributions increasing as $\frac{N !}{(N-2) ! 2 !} \sim N^{2}$. However, $W_{00}(\rho)$ decreases faster with $\rho$ than $V_{00}(\rho)$. As a result of the competition between these two different trends, the hyper-radial repulsive core appears at some $N$ growing fast with further increasing particle number so that the system is bound only due to a small attractive pocket in the asymptotic area. This is clearly seen in Fig. 3(b) for $N=200$. In this situation, the largest absolute value of nondiagonal potentials may become much larger than the depth of the attractive 

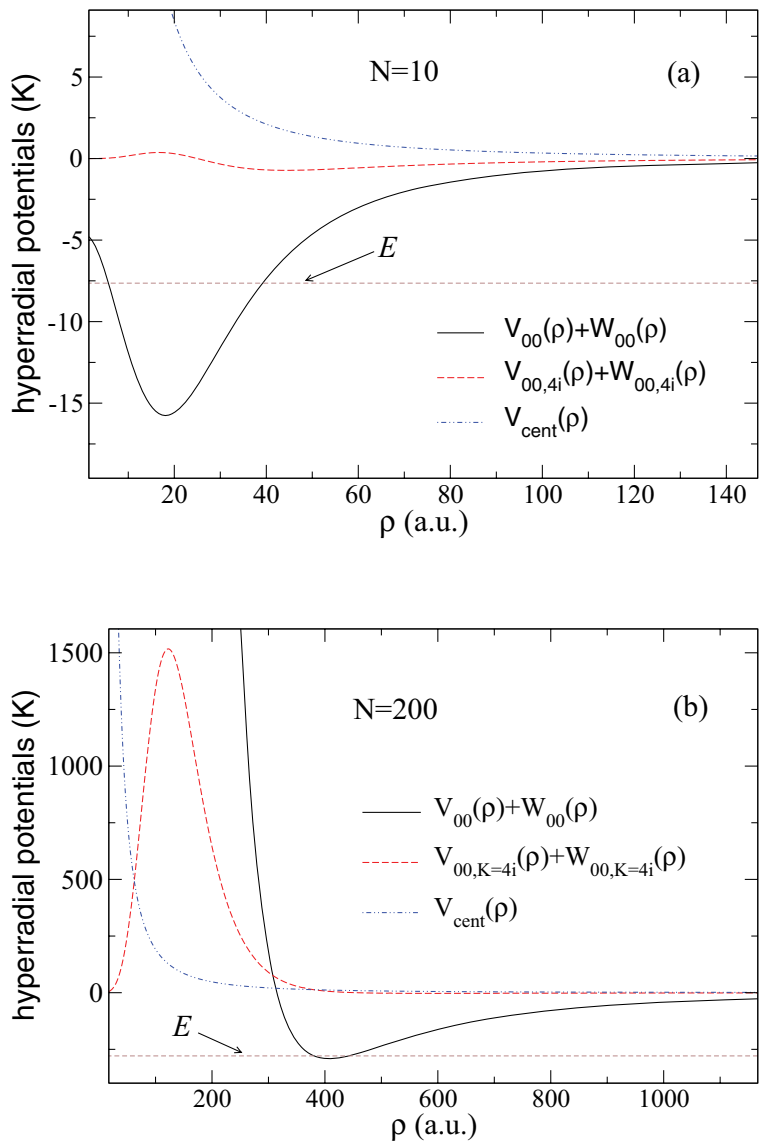

FIG. 3. (Color online) Nondiagonal hyper-radial potentials $V_{00, K=4 i}(\rho)+W_{00, K=4 i}(\rho)$ in comparison to the lowest-order potential $V_{00}(\rho)+W_{00}(\rho)$, centrifugal potential $V_{\text {cent }}(\rho)$, and the ground-state energy $E$ for $N=10$ (a) and $N=200$ (b) atoms of helium.

pocket but if the nodes of both the nondiagonal potentials and the $V_{00}(\rho)+W_{00}(\rho)$ are close to each other, the nondiagonal couplings at $\rho_{0}$ could be small with respect to the total binding energy $E$, which for large $N$ is approximately equal to the depth of the attractive pocket. This seems to be the case for $N=200$ for the particular choice of the three-body potential [see Fig. 3(b)] and a reasonably fast convergence of the hyperspherical expansion could be expected here. In general, it is difficult to make definite predictions for the binding energy convergence when three-body forces are involved because of their large hypercore. However, plotting simple graphs similar to Fig. 3 can give an idea about the convergence prior to solving the differential set of equations (4) for large $N$.

\section{DISCUSSION AND CONCLUSIONS}

It has been shown above that the convergence of the ground-state binding energy of $N$ bosons obtained from the hyperspherical-harmonics method improves with $N$ if the two-body potentials are represented by a single Gaussian. This happens because the relative strength of nondiagonal coupling potentials decreases with respect to the lowest-order diagonal potential as $N^{-1 / 2}$ or faster. This is the consequence of an increased number of exchange terms in the symmetric $N$-body wave function that give zero contribution to the nondiagonal couplings. The convergence should remain similarly good for a sum of two attractive Gaussian two-body potentials; however, it should deteriorate if one of the Gaussians is repulsive. Although individual ratios of nondiagonal to diagonal hyperradial potentials corresponding to each Gaussian decrease with $N$, the total nondiagonal potentials may not be sufficiently small at the node of the lowest-order diagonal potential or even over the range where the this potential has maximum, thus leading to a worse convergence rate. The deterioration of convergence can also be expected when a three-body hypercentral repulsive potential is included. Although the ratio of nondiagonal to diagonal hyper-radial potentials corresponding to this force decrease with $N$ in the same way as for two-body potentials (if Gaussian shape is assumed for the three-body force), the sum of the two-body and three-body contributions leads to a qualitatively new picture due to the different dependence on $N$ and $\rho$ of these contributions. At large $N$ the $N$-body systems are bound only due to a small attractive pocket in the total hyper-radial potential the depth of which (roughly equal to the total binding energy) can be very small with respect to the strength of nondiagonal couplings at their maximum. The tail of these couplings can still be strong enough at the node of the diagonal potential, thus slowing the convergence down. Whether the range of the effective three-body force can be chosen in such a way to give a good convergence over a large interval of $N$ needs further investigation.

With the range of the effective three-body force chosen in Ref. [2] for helium atoms, the $N=4,5,6$ binding energies are practically the same as those obtained in the diffusion Monte Carlo [4] and Monte Carlo hyperspherical calculations [5] with a hard-core He-He potential. On the other hand, for $N=5$ and $N=6$ the converged ground-state binding energies are very close to those obtained in the minimal approximation $K=0$. Figure 3(a) suggests that this tendency will stay at around $N=$ 10; therefore, the minimal approximation can be sufficient to calculate the ground-state energies for $7 \leqslant N \leqslant 10$ for which the diffusion [4] and hyperspherical [5] Monte Carlo calculations are available. The results of such calculations are shown in Table III. While the energy for $N=7$ is similar to exact many-body calculations with hard-core potentials, the energies for $N=8,9$, and 10 are somewhat smaller. The $K=0$ calculations have been also done for $N=20,40,70$, and 112 to compare to the Green's function Monte Carlo calculations from [11]. In this region, the $K=0$ binding energies obtained with the soft potentials are much smaller than the energies from the Green's functions Monte Carlo method with the hard core potentials. Although a noticeable contribution from $V_{00, K=4 i}(\rho)$ around the node in $V_{00}(\rho)$ is present at $20 \leqslant N \leqslant 112$ so that the binding energies will increase when other terms of the hyperspherical expansion are added, it is unlikely that this increase will be significant because $\left|V_{00, K=4 i}(\rho)\right| \ll\left|V_{00}(\rho)\right|$ for those $\rho$ where $\left|V_{00}(\rho)\right|$ has maximum.

The small binding energies for $10 \leqslant N \leqslant 112$ obtained with phenomenological potentials can be interpreted in two ways: either the effective two-body and three-body forces are $N$-dependent or a choice of the three-body repulsion is inappropriate. Although $N$-dependence of effective interactions is 
TABLE III. Absolute values of binding energies (in $\mathrm{K}$ ) of $N$ helium atoms obtained in the $K=0$ approximation ( $\mathrm{HH} 0$ ) with the phenomenological two-body soft plus three-body repulsive potential, converged hyperspherical calculations (HH) from Ref. [2] with the same potential, diffusion Monte Carlo (DMC) calculations [4], Monte Carlo hyperspherical (MCH) calculations [5], and Green's function Monte Carlo (GFMC) calculations [11] with hard-core $\mathrm{He}-\mathrm{He}$ potential. The $K=0$ calculations for a modified phenomenological three-body force (HHOM) are shown in the rightmost column.

\begin{tabular}{lcccccc}
\hline \hline$N$ & HH0 & HH & DMC & MCH & GFMC & HH0M \\
\hline 4 & 0.5389 & 0.5688 & 0.5584 & 0.5569 & 0.5334 & 0.557 \\
5 & 1.2881 & 1.3266 & 1.3022 & 1.2965 & & 1.31 \\
6 & 2.2938 & 2.3389 & 2.3194 & 2.3095 & & 2.34 \\
7 & 3.4863 & & 3.5641 & 3.5657 & & 3.58 \\
8 & 4.8040 & & 5.0374 & 5.0203 & 4.9325 & 5.02 \\
9 & 6.1995 & & 6.6765 & 6.6779 & & 6.61 \\
10 & 7.6393 & & 8.5010 & 8.4952 & & 8.35 \\
20 & 22.286 & & & & 32.546 & 31.3 \\
40 & 50.902 & & & & 99.492 & 94.9 \\
70 & 93.530 & & & & 218.68 & 213 \\
112 & 153.29 & & & & 403.2 & 402 \\
\hline \hline
\end{tabular}

a definite possibility, a different choice of phenomenological three-body force has been explored here first. It was found that for $V_{0}=-1.2343566 \mathrm{~K}$ and $\alpha=10$ a.u. from Ref. [3] and for $W_{0}=18 \mathrm{~K}$ and $\beta=5.8 \mathrm{a}$.u. the $N$-dependence of the binding energies obtained in the $K=0$ approximation is similar to that obtained with the Green's functions Monte Carlo method for $N \leqslant 112$ (see Table III and Fig. 4). Once again, these energies will increase when full hyperspherical basis is employed in the calculations. However, this simple example shows that fine tuning of the $N$-independent three-body repulsion to reproduce the binding energies of helium clusters over a wide range on $N$ is perhaps possible.



FIG. 4. (Color online) Binding energies of $N$ helium atoms obtained in $K=0$ hyperspherical-harmonics calculations with two phenomenolocial potentials, (1) $V_{0}=-1.227 \mathrm{~K}, \alpha=10.03$ a.u., $W_{0}=0.422 \mathrm{~K}, \beta=14$ a.u., and (2) $V_{0}=-1.2343566 \mathrm{~K}, \alpha=10$ a.u., $W_{0}=18 \mathrm{~K}$, and $\beta=5.8$ a.u., in comparison to the Green's function Monte Carlo calculations from Ref. [11].
Until now, only the convergence of the ground-state binding energy has been discussed. However, the system of radial equations (4) with the same hyper-radial potentials describes all excited $0^{+}$states. Since $V_{00, K i}(\rho) / V_{00}(\rho)$ for a single Gaussian potential decreases as $N^{-1 / 2}$ or faster it is expected that for a soft two-body potential the convergence of $0^{+}$ excited states improves with $N$ as well, provided these states are bound and their energy is not very much different from the ground-state energy. In fact, the calculations with the soft two-body potential from Ref. [2] for $N=10,100,200$ (not shown here) confirm this. To understand convergence for other spins and parities of the $N$-body system, the behavior of $V_{K \gamma, K^{\prime} \gamma^{\prime}}(\rho) / V_{K \gamma, K \gamma}(\rho)$ for arbitrary $K \gamma$ should be considered.

In this paper, no attempt is made to prove a decrease of $V_{K \gamma, K^{\prime} \gamma^{\prime}}(\rho) / V_{K \gamma, K \gamma}(\rho)$ with $N$ for arbitrary $K \gamma$ and arbitrary spin. Following the reasoning of Secs. III and IV may prove difficult in this case as more terms $i$ that contribute to the hyperradial potentials become available and states with other relative two-body orbital momentum should be taken into account as well. The most elegant way to proceed with analyzing the ratio $V_{K \gamma, K^{\prime} \gamma^{\prime}}(\rho) / V_{K \gamma, K \gamma}(\rho)$ in the general case would involve the fractional parentage expansion of hyperspherical harmonics introduced in Refs. [8,12]. The coefficients $a_{i}(N)$ of the present paper can, in fact, be related to the fractional expansion coefficients from these works. Investigating their behavior with increasing $N$ could be the best way to learn about convergence in the general case. Similar comments could be made about fermionic systems. It is more difficult to draw conclusions about the ratio $V_{K \gamma, K^{\prime} \gamma^{\prime}}(\rho) / V_{K \gamma, K \gamma}(\rho)$ for fermions since the minimum value of $K$ increases with $N$ due to the Pauli principle and too many $i$ 's are needed. However, one can hope that a decrease with $N$ will persist because it is related to rapid increase of the number of exchange terms in the many-body oscillator basis functions that give zero contributions to hyper-radial potentials for $K \neq K^{\prime}$.

Finally, in the present paper the convergence has been explored only for a Gaussian shape of the two-body and three-body potentials, motivated by their use in Ref. [2]. More convergence studies are needed for other shapes of the two-body and three-body potentials. However, the Gaussian shape for phenomenological effective potentials seems to be a good choice. With this choice, the binding energies for $N \leqslant 6$ helium atoms are reproduced [2] and the trend, that convergence of hyperspherical expansion for such potentials may improve with the number of particles [2], has been confirmed in the present paper. This makes the hypersphericalharmonics method with phenomenological soft two-body and repulsive three-body forces attractive for studying the properties of many-body systems in general and the universal relations in particular.

\section{ACKNOWLEDGMENTS}

The inspiration for this work has come after discussions with A. Kievsky. Support from Grant No. UK STFC $\mathrm{ST} / \mathrm{J} 000051 / 1$ is acknowledged. 
[1] A. I. Baz', Yu. T. Grin', V. F. Demin, and M. V. Zhukov, Sov. J. Part. Nuclei 3, 137 (1972).

[2] M. Gattobigio, A. Kievsky, and M. Viviani, Phys. Rev. A 84, 052503 (2011)

[3] M. Gattobigio, A. Kievsky, and M. Viviani, arXiv:1206.0854v1.

[4] M. Lewerenz, J. Chem. Phys. 106, 4596 (1997).

[5] D. Blume and C. H. Greene, J. Chem. Phys. 112, 8053 (2000).

[6] N. K. Timofeyuk, Phys. Rev. C 65, 064306 (2002).

[7] N. K. Timofeyuk, Phys. Rev. C 78, 054314 (2008).
[8] Yu. F. Smirnov and K. V. Shitikova, Sov. J. Part. Nuclei 8, 344 (1977).

[9] M. Moshinsky and Yu. F. Smirnov, The Harmonic Oscillator in Modern Physics (Harwood Academic, Amsterdam, 1996).

[10] I. J. Thompson, B. V. Danilin, V. D. Efros, J. S. Vaagen, J. M. Bang, and M. V. Zhukov, Phys. Rev. C 61, 024318 (2000).

[11] V. R. Pandharipande, J. G. Zabolitzky, S. C. Pieper, R. B. Wiringa, and U. Helmbrecht, Phys. Rev. Lett. 50, 1676 (1983).

[12] N. Barnea and A. Novoselsky, Ann. Phys. (NY) 256, 192 (1997); Phys. Rev. A 57, 48 (1998). 